LA-6073-MS

Informal Report:

\section{Higher Order Corrections to \\ Energy Levels of Muonic Atoms}

by

G. A. Rinker, Jr.

R. M. Steffen*

*Visiting Staff Member, LASL. Purdue University, Lafayette, IN 47907
UC.34a

Reporting Date: August 1975

Issued: September 1975
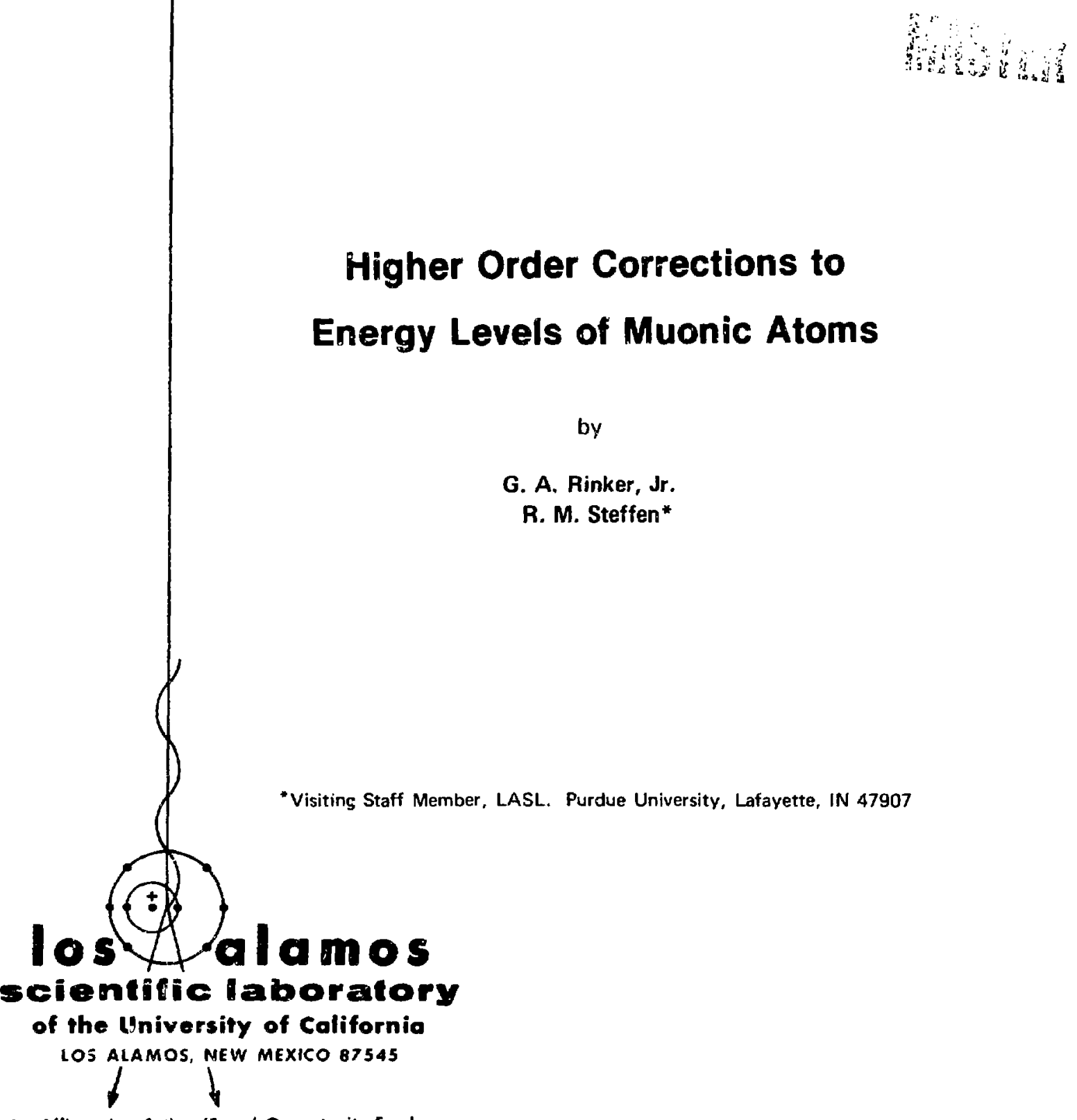

An Affirmalive Action/Equal Opportunity Employer 
In the interest of prompt distribution, this report was not edited by the Technical Information staff.

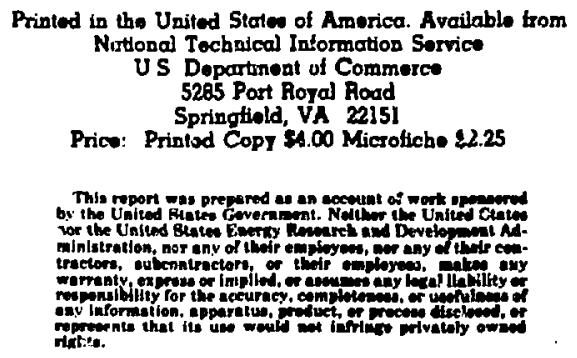


HIGHER ORDER CORRECTIONS TO ENERGY

LEVELS OF MUONIC ATOMS

by

G. A. Rinker, Jr., and R. M. Steffen

\begin{abstract}
In order to facilitate the analysis of muonic $x$-ray spectra, the results of numerical computations of all higher order quantum electrodynamical corrections to the energy levels of muonic atoms are presented in tabular and graphical form. These corrections include the vacuum polarization corrections caused by emission and re-absorption of virtual electron pairs to all orders, including "double-bubble" and "cracked-egg" diagrams. An estimate of the Delbrücke scattering-type correction is presented. The Lamb-shift (second- and fourth-order vertex) corrections have been calculated including the correction for the anomalous magnatic moment of the muon. The relativistic nuclear motion (or recoil) correction as well as the correction caused by the screening of the atomic electrons is presented in graphs. For the sake of completeness a graph of the nuclear polarization as computed on the basis of Chen's approach has been included. All calculations were made with a twoparameter Fermi distribution of the nuclear charge density.
\end{abstract}




\section{INTRODUCTION}

With presently available instrumentation the absolute energies of muonic $x$-ray transitions can be determined with a relative accuracy of about $2 \times 10^{-5}$, if adequate calibration sources are available in the energy region of interest. Before nuclear parameters can be extracted from muonic x-ray experiments, however, it is important to assess all the higher order corrections to the muonic energy levels and to be aware of the uncertainties with which these corrections can, at present, be computed.

In this work numerical values of all important energy corrections $\Delta B(n l j)$ to muonic states are presented in the form of graphs. The computer code MUON that was used for the calculations was originally written by J. G. Wills to compute muon binding energies in the field of a realistic nuclear charge distribution. The corrections discussed in this work have been incorporated into this computer program by G. A. Rinker, Jr.

The values of $\Delta B(n \ell j)$ refer to corrections of the binding energies $B(n l j)$ of the states $\mid n \ell j)$ of a muonic atom, i.e., $B(n \ell j)=m c^{2}-E(n \ell j)$, where the energies $E(n \ell j)<m c^{2}$. The notation $n l j$ refers to the description of the quantum state of the muon in the non-relativistic limit.

Transition energies (muonic $x$-ray energies) for a transition from a state $\left|n_{i} \ell_{i} j_{i}\right\rangle$ to a final state $\left|n_{f} \ell_{f} j_{f}\right\rangle$ are given by

$$
\Delta E(i \rightarrow f)=B\left(n_{f} f_{f} j_{f}\right)-B\left(n_{i} l_{i} j_{i}\right) \quad .
$$

We write the binding energy in the furm

$$
\begin{aligned}
B(n \ell j)= & B^{O}(n \ell j)+\Delta B_{V P}(n \ell j)+\Delta B_{L S^{\prime}}(n \ell j) \\
& +\Delta B_{R}(n \ell j)+\Delta B_{N P}(n \ell j)+\Delta B_{E S}(n \ell j)
\end{aligned}
$$


where $B^{\circ}(n \ell j)$ is the "uncorrected" Dirac binding energy, calculated for a bare finite-size nucleus without electrons. In all the computations a Fermi charge distribution

$$
\rho(r)=\frac{\rho_{0}}{1+\exp \left(\frac{r-c}{a}\right)}
$$

has been issumed for the nucleus with

$$
c=\left(1.183 \mathrm{~A}^{1 / 3}-0.414\right) \mathrm{fm}
$$

and

$$
a=0.55 \mathrm{fm}
$$

where $c$ is approximately the radius at which $\rho(c)=0.50 \rho(0)$ and $t=$ $(4 \ln 3) \mathrm{a}=2.417 \mathrm{fm}$ is the approximate distance over which $\rho(r)$ falls from $90 \%$ to $10 \%$ of its value at $r=0$. As compared to more realistic charge distributions, the relat: ie error of using the distribution (3) - (5) is of the order of $10^{-3}$ for the largest correction (the Uehling polarization) discussed here, and is negligibly small for the other corrections.

\section{VACUUM POLARIZATION CORRECTIONS}

The vacuum-polarization correction $\Delta B_{V P}(n l j)$ is caused by emission and re-absorption of virtual electron-positron pairs in the field of the nucleus, an effect which causes a modification of the effective electro-magnetic potential in which the muon moves (like in a dielectric medium!).

We divide the vacuum polarization into four parts:

$$
\Delta B_{V P}=\Delta B_{V P I}+\Delta B_{V P I I}+\Delta B_{V P I I I}+\Delta B_{\text {VPIV }}
$$

The four parts which will be discussed separately correspond to different types of Feynman diagrams. 


\section{$2.1 \Delta B_{V P I}(n \ell j)$, Uehling Term}

The lowest order term of this correction is represented by Diagram a), which represents the correction of order $\alpha(Z \alpha)$, or the second-order correction. [The order of the correction is defined here as the number of vertices (= powers ot $e$ in the process) minus two. The "normal" (lowest order

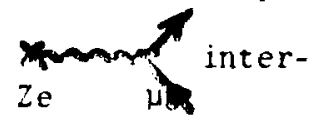
action) has two vertices and is of zero order.]

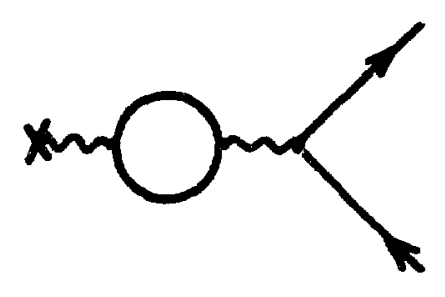

Ze

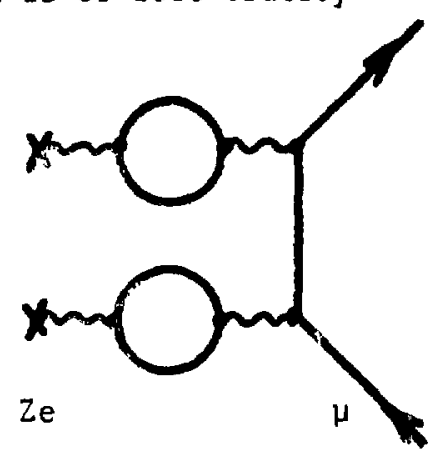

b)

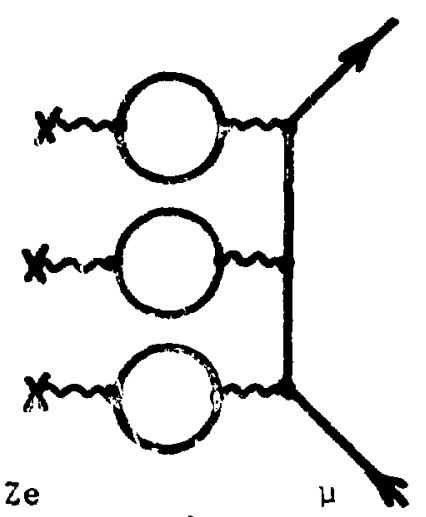

$\therefore)$

The "Uehling" vacuum polarization correction is computed by adding a vacuumpolarization potential energy $V_{V e}(r)$, the Uehling potential, to the electrostatic Coulomb energy of the nucleus ( 35 Ue, 55 Sc, 62 Fo, 68 Bar):

$$
V_{U e}(r)=+\frac{2 Z \alpha e^{2}}{3 r} \lambda_{e} \int_{0}^{\infty} \rho\left(r^{\prime}\right) r^{\prime} d r^{\prime}\left[K\left(r+r^{\prime}\right)-k\left(\left|r-r^{\prime}\right|\right)\right] \text {. }
$$

The nuclear charge distribution $\rho(r)$ is here normalized to unity $\int \rho(r) d^{3} r=1$. The function $K(x)$ is defined by the integral

$$
K(x)=\int_{1}^{\infty} \frac{\left(1+\frac{1}{2 y^{2}}\right)\left(y^{2}-1\right)^{1 / 2}}{y^{3}} e^{-2 y x / x} e d y
$$

with $*_{e}$ the reduced electron Comptor wave length. This function is evaluated as in (71 Ri). 
After adding the potential energy $V_{U e}(r)$ to the nuclear Coulomb potential the Dirac equation is solved numerically. The ladder iterations of the $\alpha$ z $\alpha$ term are included in these calculations [Diagrams b), c), etc.]. The Uehling correction $\Delta B_{V P I}(n \ell j)$, which increases the binding energy, is by far the most significant higher order correction to the binding energy of muonic states, being as large as $\Delta B_{V P I}\left(1 s_{1 / 2}\right)=+75 \mathrm{keV}$ for $z=90$.

The correction $\triangle B_{V P I}$ is relatively easy to evaluate and the numerical results are accurate within an $\mathrm{eV}$. For the analysis of muonic $x$-ray data it is necessary to compute $\triangle B_{V P I}$ for each level involved using the sarie nuclear charge distribution that is used to compute the zero-order electrostatic potentia: in the data-fitting procedure.

The Uehling vacuum-polarization correction, as conputed with the twoparameter Fermi charge distribution of Eạs. (3) - (4), is presented in Table 1 for some selected values of $Z$ and $A$.

Figure 1 shows a plot of $\Delta B_{V P I}(n \ell j)$ for the $1 s_{1 / 2}, 2 p_{1 / 2}, 2 p_{3 / 2}, 2 d_{3 / 2}$ and $4 \mathrm{f}_{5 / 2}$ mucric states as a function of $z$. The (reading) accuracy of these curves is clearly not sufficient for the analysis of muonic $x$-ray data; the curves are presented liere only as a guide.

In addition :0 the values presented in Table 1 , we have attempted to reproduce some of the $\Delta B_{V P I}(n l j)$ results of Engfer et al. (74 En), using their quoted chavge distributions. Discrepancies as large as $40 \mathrm{eV}$ were found for the low-lying muonic states of $\mathrm{Pb}$. In view of the extensive numericai checks that we made using different computational procedures, we believe that the results quoted in Table 1 are accurate to within about 1 or $2 \mathrm{eV}$ for the quoted charge distributions. 
TABLE 1

Uehling Vacuum Polarization Corrections (in eV) Computed with a Two-Paraneter Fermi Distribution of the Nuclear Charge $(c=1.183 \mathrm{Al} / 3-0.414 \mathrm{fm}, a=0.55 \mathrm{fin})$.

$\begin{array}{lllllllllll} & A & 1 s_{1 / 2} & 2 s_{1 / 2} & 2 p_{1 / 2} & 2 p_{3 / 2} & 3 d_{3 / 2} & 3 d_{5 / 2} & 4 f_{5 / 2} & 4 f_{7 / 2} & 5 g_{7 / 2}\end{array}$

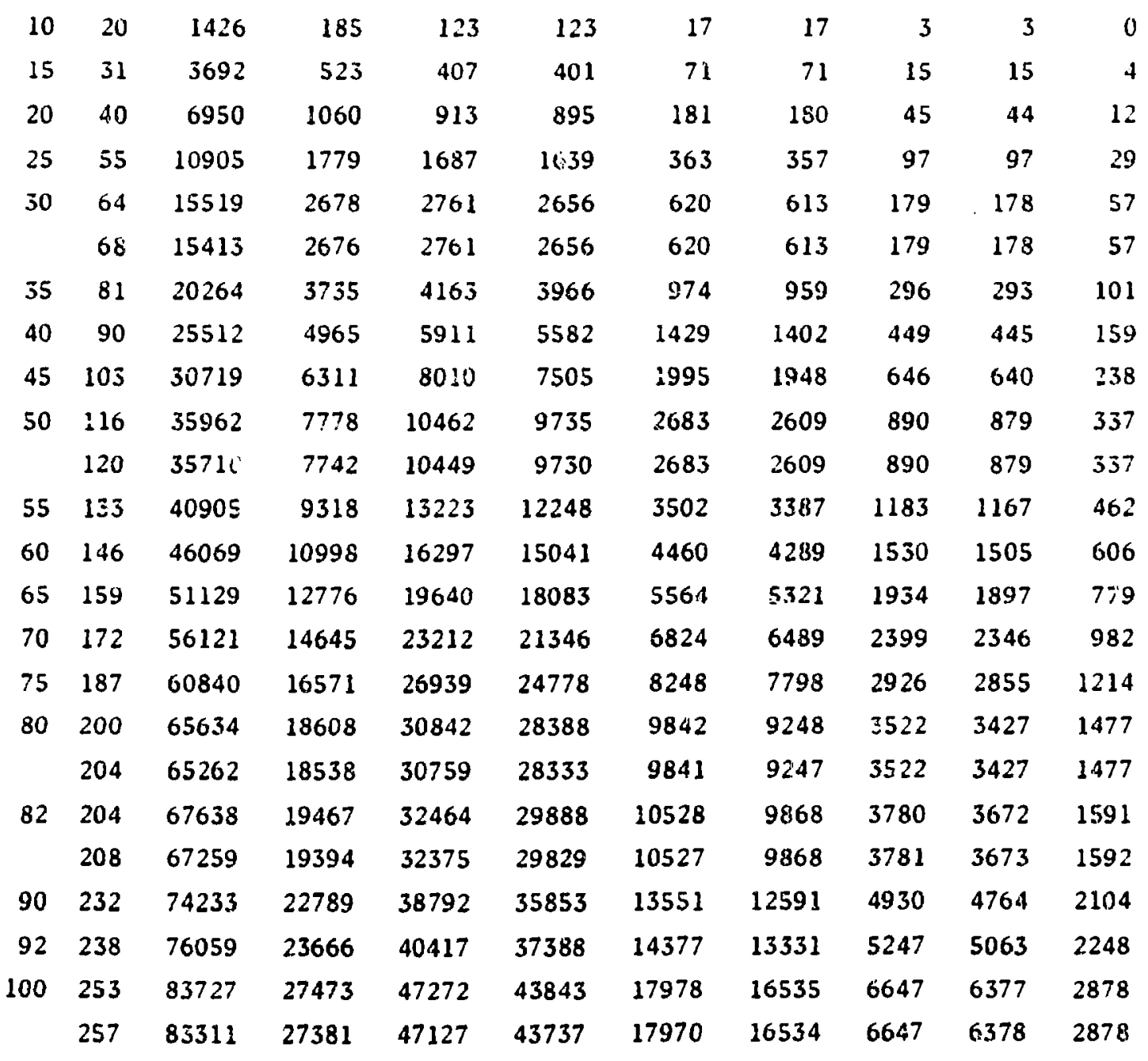




\section{$2.2 \Delta B_{V P I I}(n \ell j), K a ̈ l l e n-S a b r y$ Term}

This term is a fourth-order correction, i.e, of order $(z \alpha) \alpha^{2}$. The prescription for its calculation has first been given by Källen and Sabry (55 $\mathrm{kä).} \mathrm{The} \mathrm{term} \mathrm{consists} \mathrm{of} \mathrm{a} \mathrm{reducible} \mathrm{part} \mathrm{a)} \mathrm{("double-bubble"} \mathrm{diagram)}$ and three irreducible parts b), c), and d) ("cracked-egg diagrams").

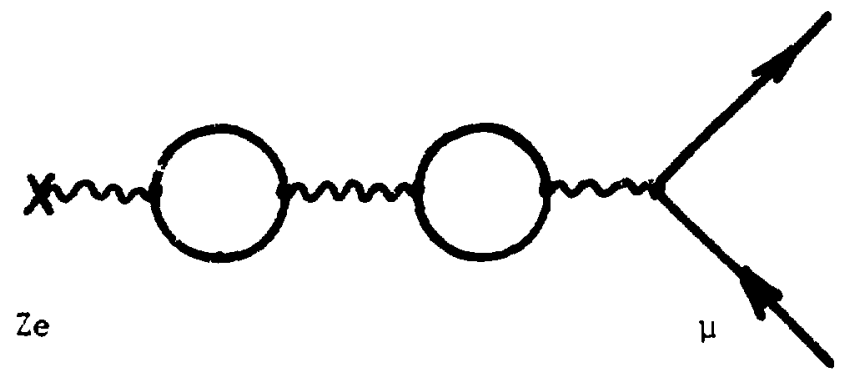

a)

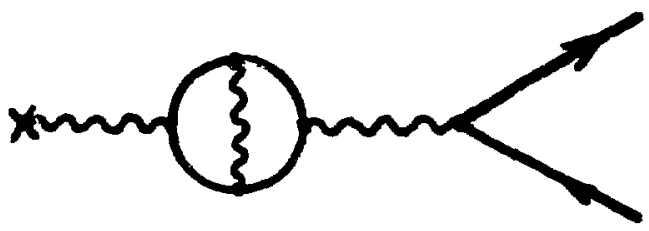

b)

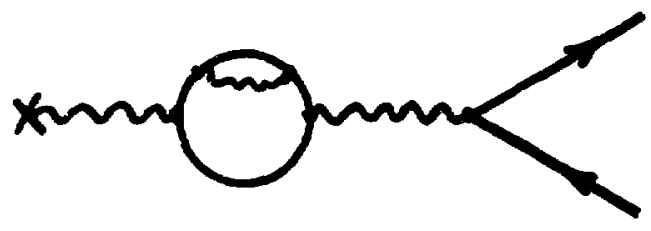

c)

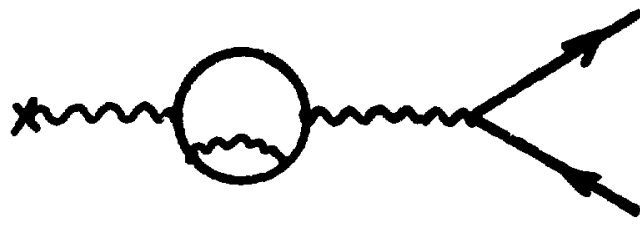

Ze

$\mu$ 
The expressions of Källen and Sabry $(55 \mathrm{Ka})$ can be reduced to a potential $V_{2 l}(r)$ (see $72 \mathrm{Bl}$ ) which must be folded with the finite charge distribution so that the finite size of the nucleus is taken into account. Figure 2 shows the "Källen" correction BVII $(n \ell j)$ calculated with the charge distribution of Eq. (3). The vacuum polarization correction $B_{V P I I}(n \ell j)$ is positive, i.e., it increases the binding energy of the muon.

$2.3 \Delta \mathrm{B}_{\mathrm{IPII}}, \alpha(Z \alpha)^{n \geqslant 3}$ term

In deriving the Uehling potential (7) it was assumed that the virtual electron and positron are propagating freely. The influence of the nuclear Coulomb field on the electron and positron wave functions can be represented by the Feynman diagrams of type:

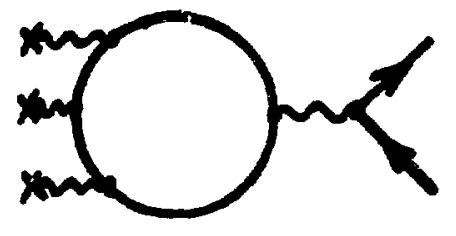

$\alpha(Z \alpha)^{3}$

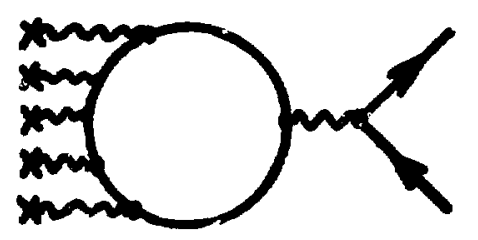

$\alpha(z \alpha)^{5}$

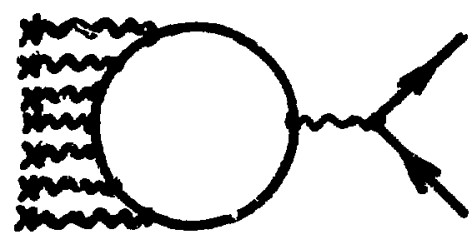

$\alpha(\mathrm{Z} \alpha)^{7}$

The distortion of the electron and positron wave functions by the nuclear Coulomb field can be interpreted in terms of a repulsion of the virtual positron and an attraction of the virtual electron which gives rise to a partial shielding of the nuclear charge and hence to a lowering of the binding energy. Hence, $\triangle B_{V P I I}$ is negative. The contribution of the diagrams $\alpha(Z \alpha)^{3}+\alpha(Z \alpha)^{5}+\alpha(Z \alpha)^{7}+\ldots$. can again be represented by the vacuun-polarization potentials $:_{13}(r)+v_{15}(r)+v_{17}(r)+\cdots \cdot=v_{l_{n}>3}(r)$. The potential $v_{1 n>3}(r)$ is repulsive for all $r$ as expected from the preceding discussion. 


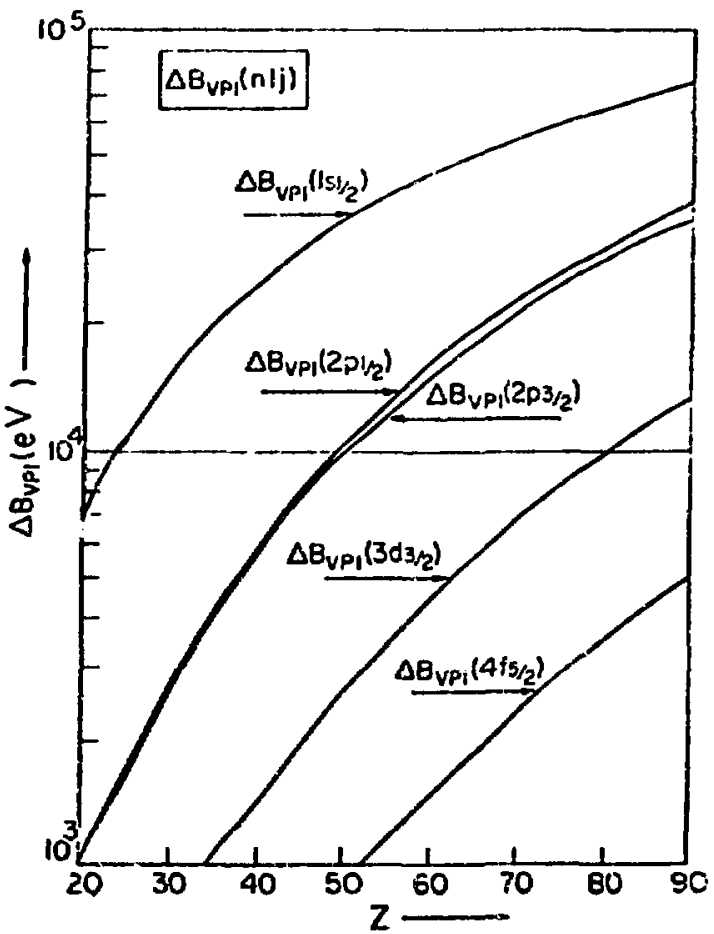

Fig. 1. "Uehling" vacuus-polarization correction.

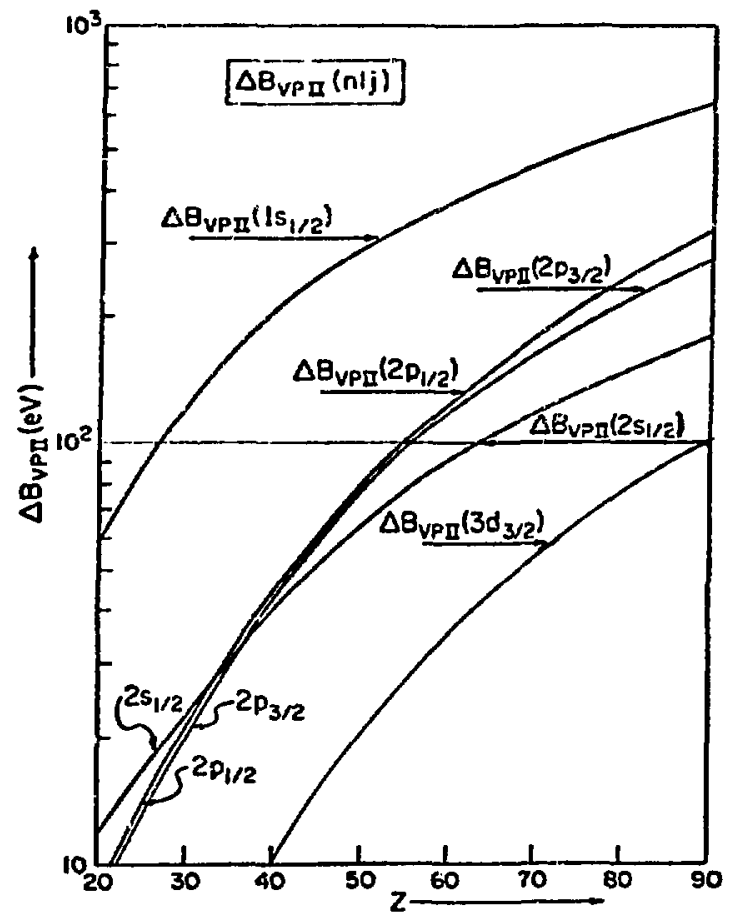

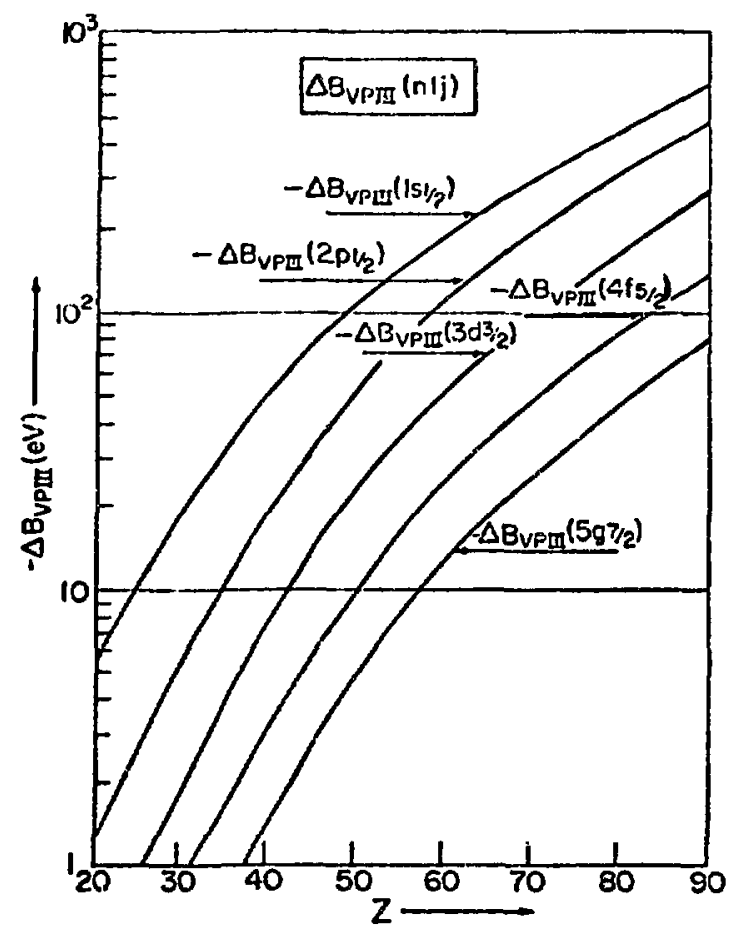

Fig. 3. $a(2 a)^{n>3}$ vacuus-polarization correction.

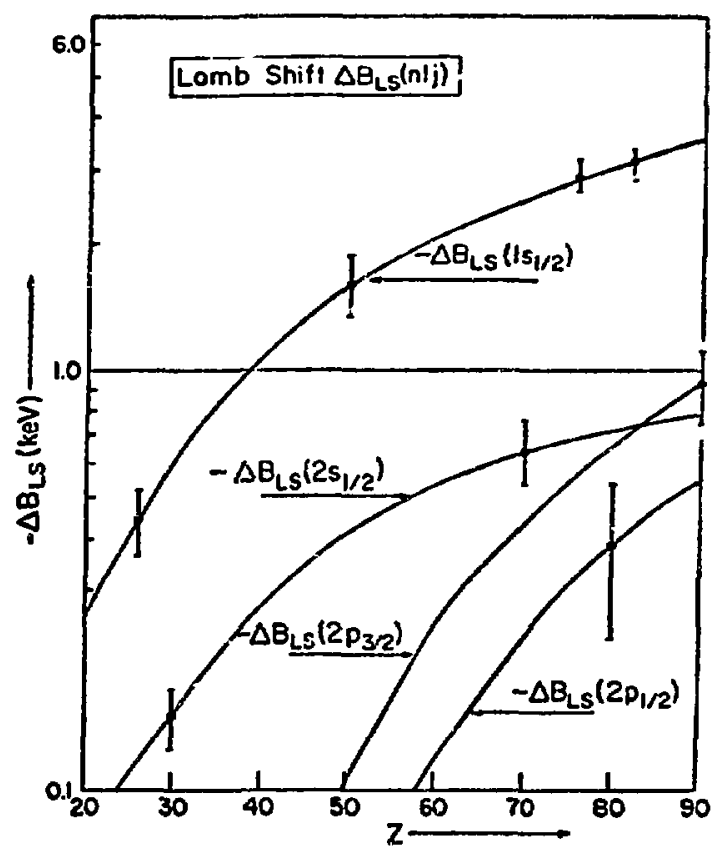

Fig. 4. "Lamb-shift" correction.

Fig. 2. "Källen-Sabry" vacuum-polmprization correction. 
The vacuum polarization correction $\Delta B_{\text {VPIII }}$ has been calculated by Rinker and Wilets (75 Ri) to all orders $n$ in $\alpha(Z \alpha)^{n}$ (n - odd) using the nuclear charge distribution (3). Figure 3 shows a plot of the results of these calculations.

2.4 The $\alpha^{2}(Z \alpha)^{2}$ Term: $\Delta B_{\text {VPIV }}(n \ell j)$

The fourth contribution to the vacuum polarization of significance, $\triangle \mathrm{B}_{V P I V}$ is of order $\alpha^{2}(Z \alpha)^{2}$ and corresponds to virtual Delbrücke scattering which is represented by the diagram

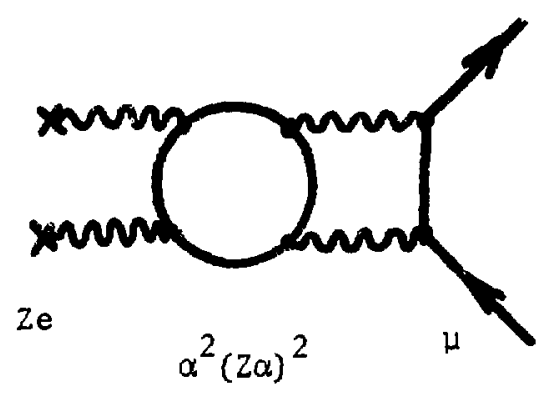

The contribution of this term has been accurately computed only for the $4 f_{5 / 2}$ state of muonic $\mathrm{Pb}$ and was found to be very small $\Delta \mathrm{B}_{\mathrm{VPIV}}\left(\mathrm{Pb} ; 4 \mathrm{f}_{5 / 2}\right)=$ $+1.0 \pm 0.1 \mathrm{eV}$ by Wilets and Rinker (75 Wi). Two subsequent calculatiuns of this term (75 Fu, $75 \mathrm{Su})$ are in good agreement with this result, while one $(75 \mathrm{Ch})$ is in substantial disagreement. There is no apparent reason to prefer the disagreeing calculation to the other three mutually independent ones, so we conclude that the Wilets and Rinker result is the correct one. A rough estimate of this small correction is provided by replacing one of the nuclear sources in the $\alpha(Z a)^{3}$ diagram by a muon source, yielding

$$
\Delta B_{V P T} V^{(n \ell j)}=-\frac{1}{2} \Delta B_{V P I I I}(n \ell j)
$$

This estimate is sufficient for muonic $x$-ray analysis, since even for the Is $_{1 / 2}$ state of muonic $U$, the correction amounts to about $\Delta B_{V P I V}\left(U, 1 s_{1 / 2}\right) \simeq$ $+7 \mathrm{eV}$, which is considerably smaller than the experimental errors. 
It is fair to say that the total vacuum polarization correction $\Delta B_{V P}(n l j)$ can at present be computed with an accuracy of a few eV.

3. THE SELF-ENERGY OR LAMB-SHIFT CORRECTION $\triangle B_{L S}(\mathbf{n} \ell j)$

We write the Lamb-Shift correction in the form

$$
\Delta B_{L S}=\Delta B_{L S I}+\Delta B_{L S I I}+\Delta B_{L S I I I}+\Delta B_{V P \mu}
$$

The main source of this correction, $\Delta B_{L S I}(n \ell j)$ is due to the virtual emission and re-absorption of photons according to the Feynman diagram

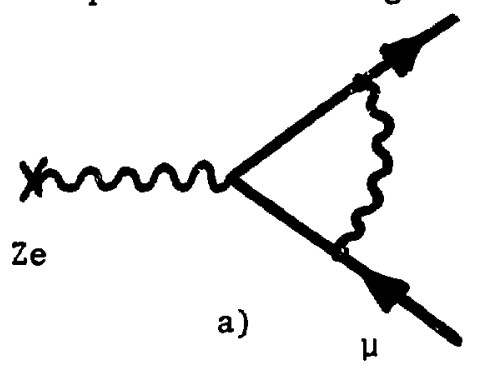

This is a second-order vertex correction of order $\alpha(z \alpha)$. In addition the (non-physical) graphs

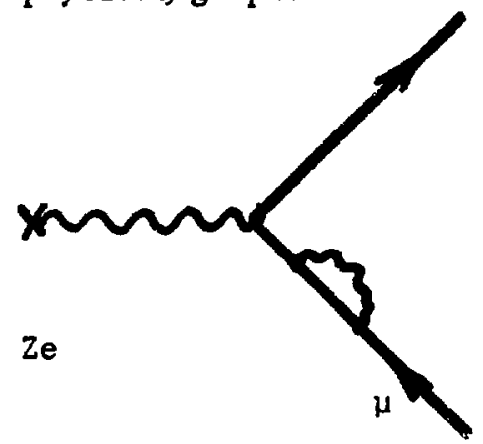

b)

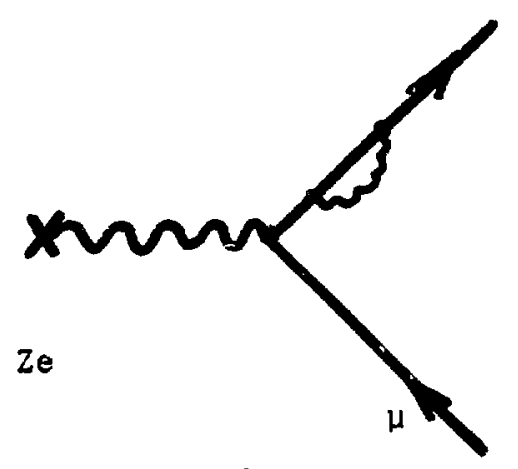

c)

contribute to the renormalization for large virtual photon momenta of graph a). The total correction $\Delta B_{L S I}(n l j)$, i.e., the second-order vertex correction of the self energy, corresponding to the lowest order diagrams is approximately (to first order in the field strength): 


$$
\begin{aligned}
\Delta B_{L S I}(n \ell j)= & -\frac{a h^{2}}{3 \pi m_{\mu}^{2} c^{2}}\left(n \ell j\left|\nabla^{2} V\right| n \ell j\right\rangle\left[\ln \left(\frac{\mu^{c}}{2 \Delta \varepsilon}\right)+\frac{11}{24}+\frac{3}{8}\right] \\
& -\frac{a h^{2}}{8 \pi m_{\mu}^{2} c^{2}}\left(n \ell j\left|\frac{2}{r} \frac{\partial V}{\partial r} \vec{\sigma} \cdot \vec{L}\right| n \ell j\right\rangle .
\end{aligned}
$$

The second term is the correction for the anomalous magnetic moment $(\alpha / 2 \pi)\left(\omega / 2 m_{\mu} c\right)$ of the muon, which couples to the magnetic field Ze $\vec{L} h / m_{\mu} c r^{3}$ as experienced by the "moving" muon. This coupling gives rise to the interaction energy

$$
\frac{z \alpha^{2} h^{3}}{4 \pi m_{\mu}^{2} c} \cdot \frac{1}{r^{3}}(\vec{L} \cdot \vec{S})
$$

The $3 / 8$ term is also due to the anomalous magnetic moment of the muon (Fermi contact interaction in s states!). These terms car be quite accurately calculated. The "Bethe Logarithm"

$$
\ln \left(\frac{m_{\mu} c^{2}}{2 \Delta \varepsilon}\right)=\frac{\sum_{\mu^{\prime}}\left\langle\mu|\vec{p}| \mu \nu\langle\mu \cdot|[V, \vec{p}]| \mu\rangle \ln \left(\frac{m_{\mu} c^{2}}{2\left|E_{\mu}-E_{\mu}\right|}\right)\right.}{\frac{\hbar^{2}}{2}\left(\mu\left|\nabla^{2} v\right| \mu\right\rangle^{2}} \text {, }
$$

where $\mid w$ are the various muonic states, is, however, cumbersome to compute with good accuracy. Barrett et al. (68 Ba) replaced $\Delta \varepsilon$ by the binding energy $B(\mu)$ of the muon state $\mid \mu)$, using $\bar{\varepsilon}=2 B(\mu)$ to produce a lower limit and $\underline{\varepsilon}=B(\mu) / 2$ to produce an upper limit of the value of the Bethe logarithm. Bethe and Negele $(68 \mathrm{Be})$ have derived rigorous upper and lower limits, which result in somewhat smaller errors than Barrett's approach. Better calculations of the Bethe logarithm which, in principle, should not present any serious difficulties, would be highly desirable for the analysis of muonic $x$-ray data. For the $1 s_{1 / 2}$ state of muonic $\mathrm{Pb}$, the correction is: $\Delta \mathrm{B}_{L S I}\left(1 \mathrm{~s}_{1 / 2}\right)=-(3.208 \pm 0.187)$ keV (Bethe-Negele limits). 
The vacuum polarization correction $\Delta B_{V P_{\mu}}(n \ell j)$ due to the creation and re-ajsorption of virtual muon pairs represented by the graph

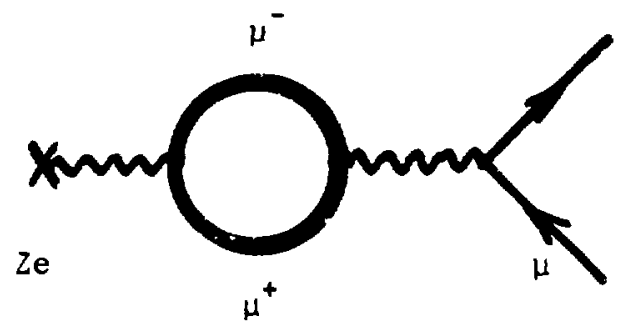

is also of order $\alpha(Z \alpha)$, and is usually included in the Lamb-shift correction for ease of computation, although it is of course a true vacuum polarization correction.

$$
\Delta B_{V P \mu}(n \ell j)=+\frac{\alpha h^{2}}{15 \pi m_{\mu}^{2} c^{2}}\left\langle n \ell j\left|\nabla^{2} V\right| n \ell j\right\rangle
$$

For the muonic $\mathrm{Pb} \mathrm{ls}_{1 / 2}$ state this correction amounts to $\Delta \mathrm{B}_{V P \mu}\left(1 \mathrm{~s}_{1 / 2}\right)=$ $+0.247 \mathrm{keV}$, with a negligible error.

In view of the experimental accuracy with which muonic $x$-ray energies can be determined today, higher order vertex corrections to the Lamb shift must be considered.

The fourth-order vertex corrections $\Delta B_{L S I I}(n \ell j)$ of order $\alpha^{2}(Z \alpha)$ that are represented by the physical diagrams

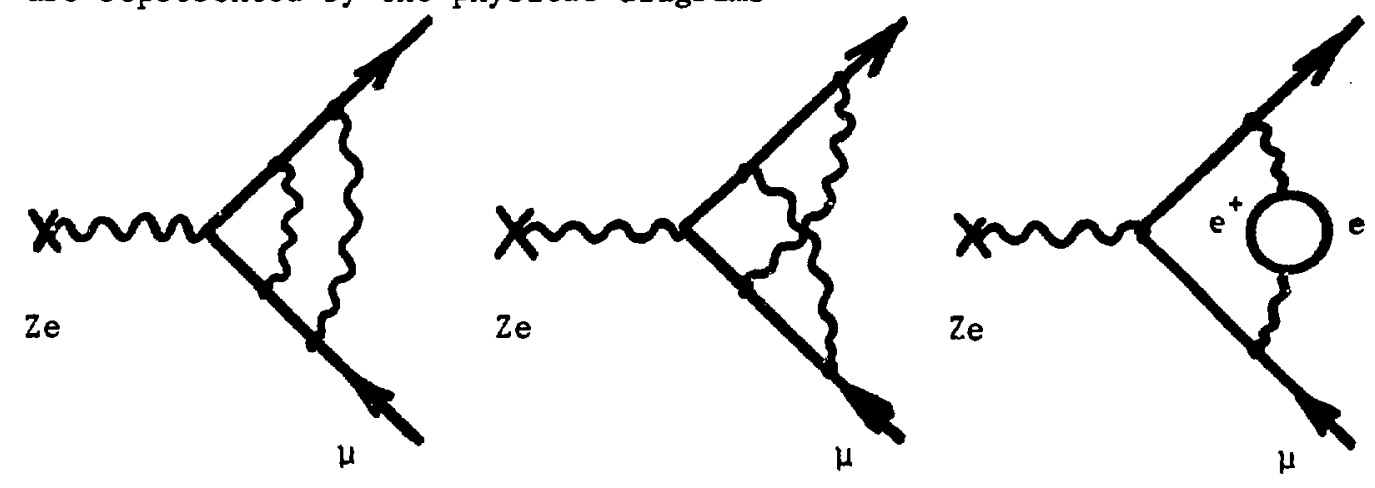



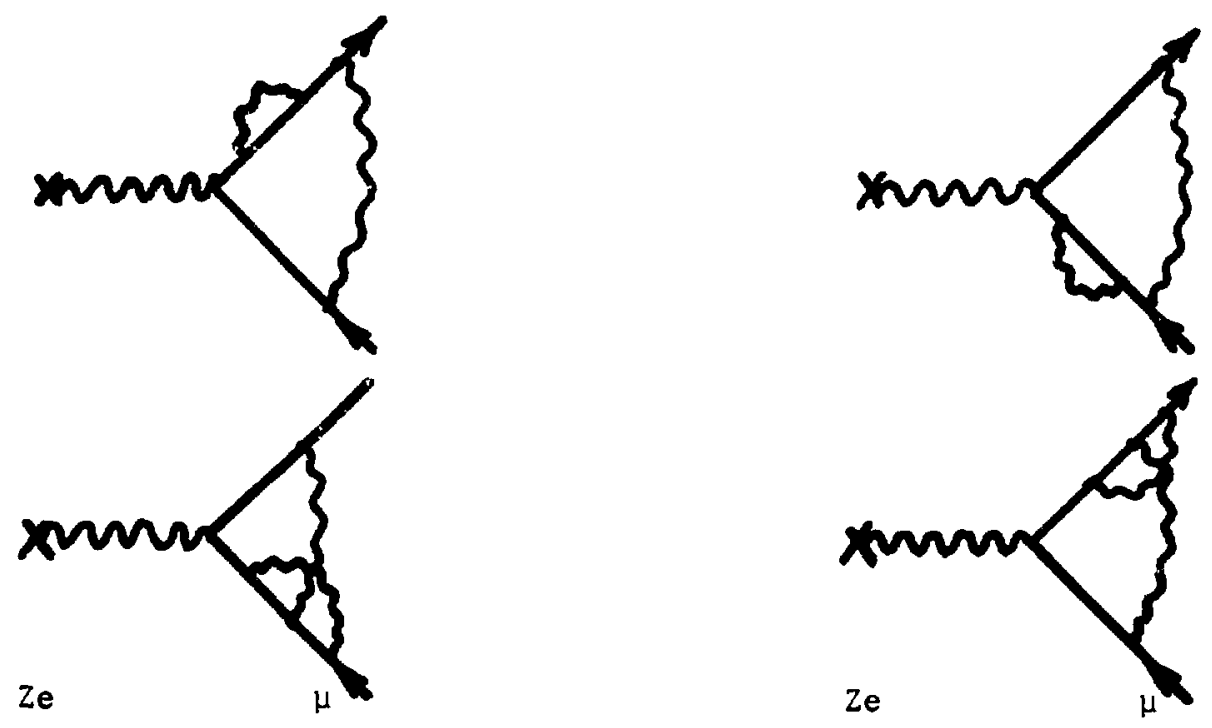

are very sinall, less than about $20 \mathrm{eV}$ for the $\mathrm{s}_{1 / 2}$ states in heavy muonic atoms. In view of the error in $\Delta B_{L S I}(n l j)$ the correction $\Delta B_{L S I I}(n l j)$ will be neglected.

On the other hand, the fourth-order vertex correction of order $\alpha(z \alpha)^{2}$, i.e., $\Delta B_{L S I I I}(n l j)$ corresponding to the diagram

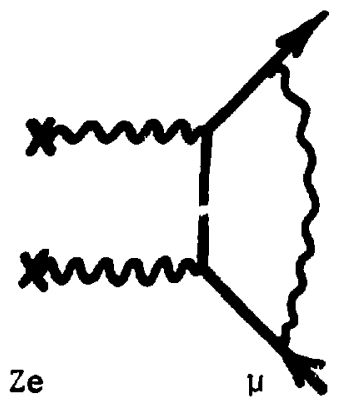

is not negligible. This term corresponds to the quadratic term in the fieid strength expansion and thus it is proportional to $\left(\varepsilon^{2}\right)$, where $\varepsilon$ is the electric field strength. The major contribution to this graph is 


$$
\Delta \max _{\operatorname{LSIII}}(n \ell j)=-\frac{20 h^{3} c}{3 \pi m_{\mu}}\left(\varepsilon^{2}\right) \int_{0}^{1} \frac{d z}{2 \min _{\mu}^{2} c^{4}+(1-z) p^{2} c^{2}} .
$$

For computational purposes, we take an average value of the local momentum over the muon wave function, i.e., $p^{2} c^{2}=\left((E-V)^{2}-m^{2} c^{4}\right)$. For muonic $\mathrm{Pb}$ the correction is $\Delta \mathrm{B}_{\mathrm{LSIII}}^{\max }\left(1 \mathrm{~s}_{1 / 2}\right)=-0.306 \mathrm{keV}$.

Since Eq. (15) represents only one contribution to this correction and cancellations usually occur, we regard it as an approximate upper limit. Hence we take

$$
\Delta B_{\text {LSI II }}(n l j) \cong\left(\frac{1}{2} \pm \frac{1}{2}\right) \Delta_{\text {LSIII }}^{\max }(n \ell j)
$$

and write for the total Lamb-shift correction

$$
\Delta B_{L S}(n \ell j)=\Delta B_{L S I}(n \ell j)+\frac{1}{2} \Delta B_{L S I I I}(n \ell j)+\Delta B_{V P \mu}(n \ell j)
$$

We assign, somewhat artificially, an error of

$$
\delta\left[\Delta B_{L S}(n \ell j)\right] \cong \pm \sqrt{\delta \Delta B_{L S I}(n \ell j)^{2}+\frac{1}{4} \Delta B_{L S I I I}^{\max }(n \ell j)^{2}}
$$

to the total Lamb-shift correction.

The total Lamb-shift correction is plotted in Fig. 4. In some points the approximate errors are indicated.

\section{RELATIVISTIC NUCLEAR MOTION (RECOIL) CORRECTION $\triangle B_{R}(n \ell j)$}

The Dirac equation is solved by using the reduced muon mass

$$
m_{\text {red }}=\frac{m_{\mu} M}{M+m_{\mu}} \text {, }
$$

where $m_{\mu}$ is the muon rest mass and $M$ is the rest mass of the nucleus. 
Relativistic effects of the nuclear motion are not taken into account by using this reduced mass.

To first order in $\beta^{2}=v^{2} / c^{2}$ and $m_{\mu} / M$, there are two major contributions to $\Delta B_{R}(n l j)$ the kinetic energy is changed because of the relativistic nuclear motion and the moving nuclear charge distribution gives rise to a retardation of the electromagnetic potential (transverse field!). The correction $\Delta B_{R}(n l j)$, which is positive, has been computed by Barrett et $a 1 .(73 \mathrm{Ba}$ ) and by Friar and Negele (73 Fr) with similar results. The relativistic nuclear motion correction $\Delta B_{R}(n l j)$ as computed with the charge distribution (3) using the Friar-Negele technique is shown in Fig. 5.

\section{ELECTRON-SCREENING CORRECTION $\Delta B_{\text {ES }}(n \ell j)$}

The energies of muonic levels are slightly shifted due to the screening of the atomic electrons. An accurate calculation of $\Delta B_{E S}(n \ell j)$ is complicated by the fact that the muon cascading down from the $n \approx 14$ level, where it is usually captured, makes predominantly Auger transitions ejecting the atomic electrons, and thus the actual populations of the electron states are uncertain. As $\mathrm{n}$ decreases the interaction of the muon with the electron structure becomes less important and radiative transitions dominate for lower n's. Fortunately, the $1 \mathrm{~s}^{2}$ electrons contribute about 90 percent of the screening and by the time it is energetically possible to eject $1 s_{1 / 2}$ electrons by Aizer transitions, the transitions are predominantly radiative Hence, in 90 percent of all cases the $1 s_{1 / 2}$ shell can be considered complete. The screening of the field, in which the atomic electrons move, by the muon must also be taken into account. Elaborate calculations have shown that it is sufficient (to within a few eV) to approximate the electron structure by that of a nucless with charge $Z-1$, so long as the muon principal quantum number $n$ is less than $\sim 10$. 


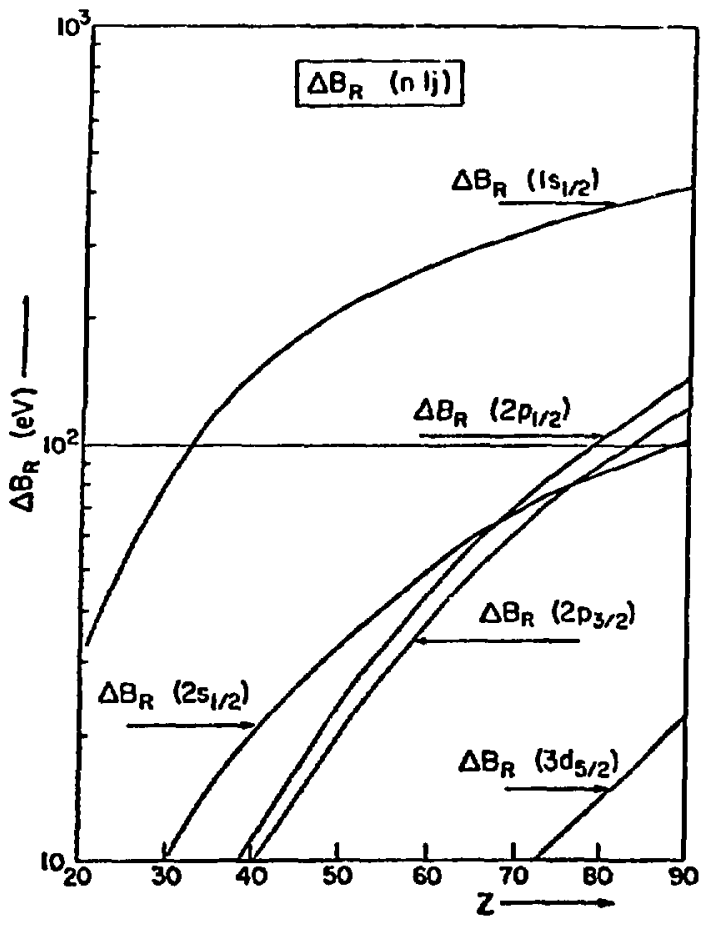

Fig. 5. Relativistic-nuclear-motion correction.

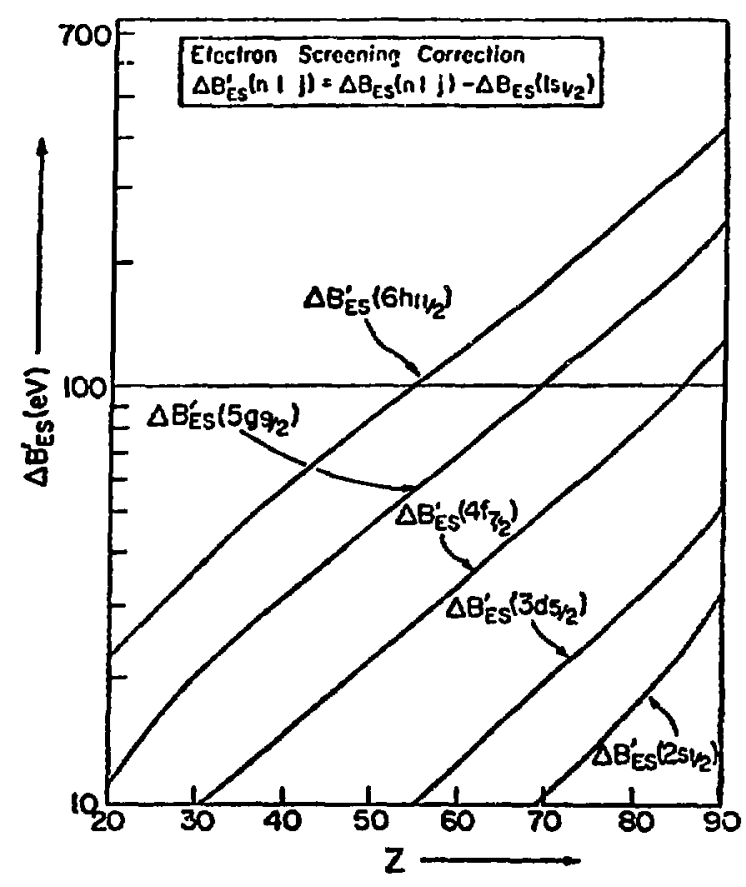

Fig. 6. Electron-sereening correction.

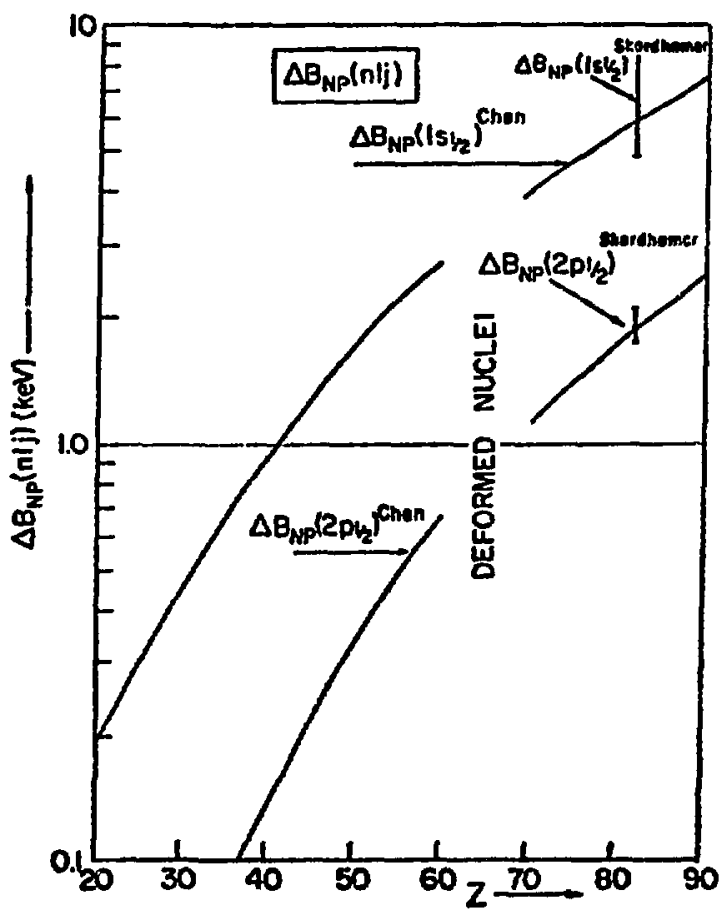

Fig. 7. Muclear-polartzation correction. 
In order to account for the screening of the atomic electrons a screening potential $V_{s c r}(r)$ may be added to the nuclear Coulomb potential and the vacuum polarization potentials, before the Dirac equation for the muon is solved. The screening potential energy is

$$
V_{s c r}(r)=\frac{4 \pi e^{2}}{r} \int_{0}^{r} \rho_{e l}(t) t^{2} d t+4 \pi e^{2} \int_{r}^{\infty} \rho_{e l}(t) t d t
$$

where $\rho_{e l}(r)$ is the spherically averaged electron density of the atom, normalized to $4 \pi \int_{0}^{\infty} \rho_{e l}(r) r^{2} d r=$ number of electrons $(73 \mathrm{Vo})$.

The second term of $\mathrm{Eq}$. (20) gives rise to a potential at $r=0$ of the order of $V_{s c r}(0) \approx 0.0492^{4 / 3} \mathrm{keV}$. At larger radii $r, v_{s c r}(r)$ is a slowly decreasing function of $r$. Hence, $\left|\Delta B_{E S}(n l j)\right|$ is smaller for large values of $n$, the largest correction occuring in the $1 s_{1 / 2}$ state $\left[\Delta B_{E S}(n \ell j)\right]$. Thus the quantity

$$
\Delta B_{E S}^{\prime}(n \ell j)=\Delta B_{E S}(n \ell j)-\Delta B_{E S}\left(1 s_{1 / 2}\right)
$$

is always positive (or zeroj). In other words, the electron-screening correction reduces the transition energies of the muonic $x$ rays [see Eq. (1)].

Since only transition energies between muonic levels are observed, it is sufficient to know the correctjon $\Delta B^{\prime}{ }_{E S}(n l j)$ of Eq. (21). Values of $\Delta B^{\prime}(n \& j)$ have been computed with the charge distribution (3) and for a complete atoinic electron shell, using true Hartree-Fock electron densities (75 Ma). The results are shown in Fig. 6.

\section{NUCLEAR POLARIZATION CORRECTION BNp(nlj)}

So far it has been assumed that the muon moves in the static Coulomb field of the nucleus and the various higher order corrections were taken 
into account assuming a static field produced by an inert nucleus. In other words, it was assumed that the nucleus is not affected by the presence of the muon.

The nucleus: however, is not an inert structure; it is a complicated system of protons and neutrons with a large number of excitation modes. For instance, the muon charge density penetrates into the nuclear volume thus reducing the Coulomb repulsion inside the nucleus.

Classically speaking, this effect causes a shrinking of the nucleus and hence the binding energies of the muonic levels are increased. Quantummechanically speaking, the electromagnetic interaction between the muon and the nucleons in the nucleus induces virtual transitions to excited intermediate states of the muon-nucleus system. Obviously, an exact calculation of the nuclear polarization requires knowledge of the complete spectrum of excited nuclear states and their transition strengths, information which, in general, is not available.

The Hamiltonian for the muon-nucleus system can be expressed in the form

$$
H=H_{\text {Nuc leus }}^{0}+H_{\mu}^{0}+H_{\mathrm{N} \mu},
$$

where $H_{\text {Nucleus }}^{0}$ is the Hamiltonian of the isolated nucleus; $H_{\mu}^{0}$ is the Hamiltonian of the free muon and $\mathrm{H}_{\mathrm{N \mu}}$ describes the electromagnetic interaction between the nucleus and the muon, i.e.,

$$
H_{N \mu}=\int d^{3} \vec{r}_{N} \frac{\rho_{o p}\left(\vec{r}_{N}\right)}{\left|\vec{x}_{\mu}-\vec{r}_{N}\right|} \text {, }
$$


where $\rho_{\text {op }}\left(\vec{r}_{N}\right)$ is the one-body charge density operator for the nucleus. The expectation value of $\rho_{o p}\left(\vec{r}_{N}\right)$ in any nuclear state gives the electrostatic nuclear charge distribution for that state. It is convenient to write the interaction Hamiltonian in the form

$$
H_{\mathrm{N} \mu}=V\left(\overrightarrow{\mathrm{r}}_{\mu}\right)+\delta \mathrm{H}_{\mathrm{N} \mu}
$$

where

$$
V\left(\overrightarrow{\mathbf{r}}_{\mu}\right)=\left\langle\left. 0\right|_{\mathrm{N}_{\mu}} \mid 0\right\rangle
$$

is the expectation value of $\mathrm{H}_{\mathrm{N} \mu}$ in the nuclear ground state. With the definitions of $\delta \mathrm{H}_{N \mu}$ by Eqs. (24) and (25), $\delta \mathrm{H}_{N \mu}$ gives no contribution to the energy in first-order perturbation theory. We expand $\delta_{\mathrm{N \mu}}$ in terms of multipole operators

$$
\delta H_{N \mu}=\sum_{L=0}^{\infty} \delta H_{L}
$$

with

$$
\delta H_{L}=\int d^{3} \vec{r}_{N} \rho_{O p}\left(\vec{r}_{N}\right) \sum_{M} \frac{4 \pi}{2 L+1} \frac{r_{L}^{L}}{r_{>}^{L+1}} Y_{L M}^{*}\left(S_{N}\right) Y_{L M}\left(\Omega_{\mu}\right)-V\left(\vec{r}_{\mu}\right)
$$

Since $V\left(\vec{r}_{\mu}\right)$ contains no nuclear coordinates, it does not contribute to transition matrix elements between different nuclear states and it is usually discarded after noting that $\left(0\left|\delta H_{L}\right| 0\right\rangle=0$, where $|0\rangle$ is the nuclear ground state.

The second-order correction to the energy $E(n l j)$ of the muonic atom in the state $|0 ; n \ell j|$ is given by 


$$
\Delta E_{N P}(n \ell j)=\sum_{L, I \omega, n^{\prime} \ell^{\prime} j^{\prime}} \frac{\left\langle 0 ; n \ell j\left|\delta H_{L}\right| I \omega ; n^{\prime} \ell^{\prime} j^{\prime}\right)\left\langle I \omega ; n^{\prime} \ell^{\prime} j^{\prime}\left|\delta H_{L}\right| 0 ; n \ell j\right\rangle}{E_{0}+E(n \ell j)-E_{I \omega}-E\left(n^{\prime} \ell^{\prime} j^{\prime}\right)}
$$

where $I \omega$ are the quantum numbers of the nuclear states. The sum over the nuclear states $|I \omega\rangle$ excludes the nuclear ground state $\mid 0)$.

The $L=O$ monopole term, $\delta H_{0}$, depends on the excitation energy of the, in general, unobserved, monopole excitations (breathing modes) of the nucleus. Because of the limited information on nuclear compressibility, etc., only an approximate calculation of this term is possible. The $L=1$ or dipole term $\delta \mathrm{H}_{1}$ depends on the, in general, known energy and strength of the giant-dipole term resonance state. For $L>2$ the results are rather insensitive to the energies of the nuclear states involved. As an order of magnitude illustration the contributions to the nuclear polarization correction of the $1 s_{1 / 2}$ states of muonic ${ }^{208} \mathrm{~Pb}$ as computed by Skardhamar $(70 \mathrm{Sk})$ are given: $\Delta \mathrm{B}_{\mathrm{NP}}\left(1 \mathrm{~s}_{1 / 2}\right.$, monopole breathing mode $)=2.1 \mathrm{keV}, \Delta \mathrm{B}_{\mathrm{NP}}\left(1 \mathrm{~s}_{1 / 2}\right.$, isovector dipole $)=1.7 \mathrm{keV}, \Delta \mathrm{B}_{\mathrm{NP}}\left(1 \mathrm{~s}_{1 / 2}\right.$, quadrupole and octupole shape vibrations) $=1.1 \mathrm{keV}$.

Nuclear polarization corrections have been calculated by Cole (69 Co), Chen (70 Ch, $70 \mathrm{Che})$ and Skardhamar (70 Sk). The results of some of these calculations are shown in Fig. 7. The curves are only intended as a rough guide; the uncertainty of $\Delta B_{N P}$ is at least 30 percent.

The nuclear polarization correction for deformed nuclei requires a more refined procedure than outlined above. The nuclear polarization correction is obtained by extending the "model space" [usually spanned by the lowest-rotational-band states of the nucleus and the muon spin multiplet wave-functions $1 s_{1 / 2},\left(2 p_{1 / 2}, 2 p_{3 / 2}\right),\left(3 d_{3 / 2}, 3 d_{5 / 2}\right)$ etc.] to include configurations up to several MeV excitation energy. This leads to a renormalization 
of the intrinsic quadrupole moment $Q_{0}$ of the nuclear state and to a constant energy shift $\Delta E$ of the hyperfine components of a "model space" state, when a nonrelativistic treatment is used $(70 \mathrm{Che})$. This energy shift $\triangle \mathrm{E}$ corresponds to the nuclear polarization correction $-\Delta B_{N P}(n l j)$.

For a reliable analysis of muonic $x$-ray data, the nuclear polarization correction must be computed for each nucleus individually. 


\section{REFERENCES}

35 Ue E. A. Uehling, Phys. Rev. 48, 55 (1935).

55 Kä B. Källen and A. Sabry, Dan. Met. Fys. Medd. 29, No. 17 (1955).

55 Sc J. Schwinger, Phys. Rev. 75, 651 (1955).

62 to K. W. Ford and J. G. Wills, Nuel, Piys.,., 295 (1962)

68 Bar Barrett et al., Phys. Rev. 166, 1589 (1968).

$68 \mathrm{Ba}$ R. C. Barrett, Phys. Lett. 28B, 93 (1968).

$68 \mathrm{Be}$ H. A. Bethe and J. W. Negele, Mucl. Phys. A117, 575 (1968).

69 Co R. K. Cole, Phys. Rev. 177, 164 (1969).

$70 \mathrm{Ch}$ Min-Yi Chen, Phys. Rev. Cl, 1167 (1970).

70 Che Min-Yi Chen, Phys. Rev. C1, 1176 (1970).

$70 \mathrm{Sk}$ H. F. Skardhamar, Nucl. Phys. A151, 154 (1970).

71 Ri G. A. Rinker, Jr., Ph.D. Thesis, University of California, Irvine, CA, (unpublished).

72 Bl J. Blomqvist, Nuc1. Phys. B48, 95 (1972).

$73 \mathrm{Ba}$ Barrett et al., Phys. Lett. 47B, 297 (1973).

73 Fr J. L. Friar and J. W. Negele, Phys. Lett. 46B, 5, (1973).

73 Vo P. Voge1, Phys. Rev. 7A, 63 (1973).

74 En R. Engfer et al., Atomic and Nuc1. Data Tables 14, 509 (1974).

$75 \mathrm{Fu}$ D. Fujimoto, to be published.

$75 \mathrm{Su}$ M. K. Sundarisan and P. J. S. Watson, Int. Conference in High-Energy Physics and Nuclear Structure, Santa Fe, NM, June 1975.

75 Ch Min-Yi Chen, Phys. Rev. Lett. 34, 341 (1975).

$75 \mathrm{Ri}$ G. A. Rinker and L. Wilets, Phys. Rev., to be published.

75 Wi L. Wilets and G. A. Rinker, Phys. Rev. Lett. 34, 339 (1975).

$75 \mathrm{Ma}$ J. B. Mann and G. A. Rinker, Jr., Phys. Rev. All, 385 (1975). 